\title{
THE LEAST SQUARES STOCHASTIC FINITE ELEMENT METHOD IN STRUCTURAL STABILITY ANALYSIS OF STEEL SKELETAL STRUCTURES
}

\author{
M. KAMIŃSKI* and J. SZAFRAN \\ Department of Structural Mechanics \\ Faculty of Civil Engineering \\ Architecture and Environmental Engineering \\ Al. Politechniki 6, 90-924 Łódź, POLAND \\ E-mail: Marcin.Kaminski@p.lodz.pl \\ Jacek.Szafran@p.lodz.pl
}

\begin{abstract}
The main purpose of this work is to verify the influence of the weighting procedure in the Least Squares Method on the probabilistic moments resulting from the stability analysis of steel skeletal structures. We discuss this issue also in the context of the geometrical nonlinearity appearing in the Stochastic Finite Element Method equations for the stability analysis and preservation of the Gaussian probability density function employed to model the Young modulus of a structural steel in this problem. The weighting procedure itself (with both triangular and Dirac-type) shows rather marginal influence on all probabilistic coefficients under consideration. This hybrid stochastic computational technique consisting of the FEM and computer algebra systems (ROBOT and MAPLE packages) may be used for analogous nonlinear analyses in structural reliability assessment.
\end{abstract}

Key words: weighted least squares method, stochastic finite element method, generalized stochastic perturbation technique, stability problems, steel skeletal structures.

\section{Introduction}

One of the most challenging problems in the design of skeletal structures is a demand for an extra uploading with the additional technological equipment, maybe far beyond the design limits given in the initial structural project. As far as the steel or related thin-walled structures are concerned, verification of the capacity margin must be complemented each time with the stability analysis taking into account both global as well as local instabilities. Computational analysis provided with the use of the Finite Element Method (FEM) or some of its stochastic counterparts should account for all the external loads, where the technological one is a subject of an incrementation procedure in our model leading in turn to a determination of the critical load multipliers. It is usually done by means of the linearized buckling FEM analysis, where constitutive, initial stress and initial displacements stiffness matrices compose the overall engineering structure stiffness. On the other hand, the uncertainty sources appear as geometrical imperfections, stochastic decreases of the given cross-sections due to corrosion (Melchers, 1987; Sadovský and Drdácký, 2001) and/or environmental external loadings as ice covers or wind pressures. They all influence the reliability index calculated according to the first (FORM) or the second order reliability methods (SORM), where the limit function is a difference between computed normal stresses and these corresponding to the stability limit. Some other issues connected with uncertainty analysis, particularly in stability verification, may be found in Elishakoff (1983), Elishakoff (2000), Elishakoff et al. (2001).

\footnotetext{
* To whom correspondence should be addressed
} 
Therefore, the main purpose of this work it to show the Weighted Least Squares Method approach to the perturbation-based Stochastic Finite Element Method (Kamiński, 2013; Kamiński and Solecka, 2013; Kamiński and Strąkowski, 2013) being a generalization of the Second Order Second Moment (SOSM) stochastic perturbation approach (Kleiber and Hien, 1992). Particularly, we demonstrate its application to the stability analysis of the steel skeletal structures (towers) to verify an opportunity to upload them significantly beyond initial designing limits in the presence of some random parameters. This analysis is carried out for the steel telecommunication tower having Young modulus randomized according to the Gaussian probability distribution function, rectangular cross-section with linear geometrical convergence to the highest segment and subjected to the wind blow as well as dead, technological and live loads. We provide the critical load as the unit vector acting downwards and placed at the tower top, which simulates the capacity margin for this structure and accounting for the extra equipment to be mounted for the future telecommunication applications. Considering the statements of Eurocode 0 we need to verify also whether the critical load magnitude is the Gaussian variable - to use further the simplified reliability index formula that consists of a ratio of the expectations to the standard deviations of the limit function. It should be underlined that this critical load magnitude is automatically a limit function itself in our model, so that the output coefficient of variation is an inverse of the reliability index according to the Cornell theory (Melchers, 1987). We examine convergence of all the probabilistic characteristics examined together with the order of stochastic perturbation method applied as well as with the distribution of the weights inside a computational domain; all as the functions of input coefficient of variation and approximating polynomial order. The general observation is that we get the distribution of the critical force close enough to the Gaussian one and stable values of all the computed quantities for an input coefficient smaller or equal to 0.10 . Finally, we notice a linear interrelation in-between the output and input uncertainty sources, so the random dispersion level is maintained during stability problem solution including even all geometrical nonlinearities in a buckling phenomenon. Considering the above it seems that the general idea to calculate the critical force from an algebraic relation close to the Euler one (Timoshenko and Gere, 1961), where the critical load magnitude is proportional to the initial Young modulus of the structural steel, is quite well justified in the steel skeletal structures (Elishakoff, 2000; Kamiński and Strąkowski, 2013).

\section{Governing variational equations}

Let us consider a statistically homogeneous and bounded region $\Omega \subset \mathfrak{R}^{3}$ with no initial stresses and strains. Elastic properties and geometry of $\Omega$ may be treated as design random parameters and they result in the random displacement field and random stress tensor satisfying the boundary-value problem of linear elasticity given below. Let us assume that there are non-empty subsets of external boundaries $\partial \Omega_{\sigma}$ and $\partial \Omega_{u}$ of $\Omega$, where the stress and displacement boundary conditions are defined. The elasticity equilibrium problem can be proposed as

$$
\begin{aligned}
& \sigma_{i j}=C_{i j k l} \varepsilon_{k l}, \\
& \varepsilon_{i j}=\frac{1}{2}\left(u_{i, j}+u_{j, i}\right), \\
& \sigma_{i j, j}+\rho f_{i}=0, \\
& u_{i}=\hat{u}_{i} ; \quad x_{i} \in \partial \Omega_{u}, \\
& \sigma_{i j} n_{j}=\hat{t}_{i} ; \quad x_{i} \in \partial \Omega_{\sigma},
\end{aligned}
$$


where

$$
C_{i j k l}=\delta_{i j} \delta_{k l} \frac{e v}{(1+v)(1-2 v)}+\left(\delta_{i k} \delta_{j l}+\delta_{i l} \delta_{j k}\right) \frac{e}{2(1+v)}
$$

for $i, j, k, l=1,2,3$. Let us note that theoretical foundations and some numerical aspects of elliptic boundary value problems with random coefficients are proposed by Kleiber and Hien (1992). This system is generalized in our study to the following incremental form:

$$
\begin{aligned}
& \Delta \sigma_{k l, l}+\rho \Delta f_{k}=0 ; \quad x_{i} \in \Omega, \\
& \Delta \tilde{\sigma}_{k l}=C_{k l m n} \Delta \varepsilon_{m n} ; \quad x_{i} \in \Omega, \\
& \Delta \varepsilon_{m n}=\frac{1}{2}\left(\Delta u_{m, n}+\Delta u_{n, m}+u_{i, m} \Delta u_{i, n}+\Delta u_{i, m} u_{i, n}+\Delta u_{i, m} u_{i, n}\right) ; \quad x_{i} \in \Omega,
\end{aligned}
$$

with the following incremental boundary conditions:

$$
\begin{array}{ll}
\Delta \sigma_{k l} n_{l}=\Delta \hat{t}_{k} ; & x_{i} \in \partial \Omega_{\sigma}, \quad k=1,2,3, \\
\Delta u_{k}=\Delta \hat{u}_{k} ; & x_{i} \in \partial \Omega_{u}, \quad k=1,2,3 .
\end{array}
$$

This problem is also solved for the overall displacement vector $u_{k}(\boldsymbol{x})$, the strain tensor $\varepsilon_{k l}(\boldsymbol{x})$ and the stress tensor $\sigma_{k l}(\boldsymbol{x})$, where the tensor functions $\Delta \sigma_{k l}(\boldsymbol{x}), \Delta \tilde{\sigma}_{k l}(\boldsymbol{x})$ are the first and the second PiolaKirchhoff tensors

$$
\Delta \sigma_{k l}=\Delta F_{k m} \Delta \tilde{\sigma}_{m l}+F_{k m} \Delta \tilde{\sigma}_{m l}+\Delta F_{k m} \tilde{\sigma}_{m l} ; x_{i} \in \Omega
$$

and where

$$
\Delta F_{k m}=\Delta u_{k, m} ; x_{i} \in \Omega
$$

The following functional defined on $\Delta u_{k}$ is introduced in order to obtain an additional variational formulation:

$$
J\left(\Delta u_{k}\right)=\int_{\Omega}\left(\frac{1}{2} C_{k l m n} \Delta \varepsilon_{k l} \Delta \varepsilon_{m n}+\frac{1}{2} \tilde{\sigma}_{k l} \Delta u_{i, k} \Delta u_{i, l}-\rho \Delta f_{k} \Delta u_{k}\right) d \Omega-\int_{\partial \Omega} \Delta \hat{t}_{k} \Delta u_{k} d(\partial \Omega) .
$$

Its minimization with respect to this $\Delta u_{k}$ leads to the Finite Element Method equations described in details in the next section. 


\section{Stochastic Finite Element Method equations}

In the FEM approach we adopt the following well known discretization of the displacement function increments in the RFM analysis (Kamiński, 2013; Kamiński and Solecka, 2013; Kamiński and Strąkowski, 2013):

$$
\Delta u_{\alpha}=\phi_{\alpha \beta} \Delta q_{\beta}=\phi_{\alpha \beta} D_{\beta}^{(p)} b^{p}, \quad p=0, \ldots, n-1 ; \quad \beta, \alpha=1, \ldots, N
$$

We rewrite additionally strain tensor components discretization as

$$
\Delta \varepsilon_{k l}=B_{k l \beta} \Delta q_{\beta}=B_{k l \beta} D_{\beta}^{p} h^{p}, \quad p=0, \ldots, n-1 ; \quad \beta=1, \ldots, N ; \quad k, l=1,2,3
$$

and the stress tensor components

$$
\begin{aligned}
& \Delta \sigma_{i j}=C_{i j k l} \Delta \varepsilon_{k l}=C_{i j k l} B_{k l \beta} \Delta q_{\beta}=C_{i j k l} B_{k l \beta} D_{\beta}^{p} h^{p}, \\
& p=0, \ldots, n-1 ; \quad \alpha, \beta=1, \ldots, N ; \quad i, j, k, l=1,2,3 .
\end{aligned}
$$

Inserting these statements into the geometrical and constitutive equations one may obtain the functional given above as the one proposed by Kleiber (1985).

$$
J\left(\Delta q_{\alpha}\right)=\frac{1}{2} K_{\alpha \beta}^{(1)} \Delta q_{\alpha} \Delta q_{\beta}+\frac{1}{3} K_{\alpha \beta \gamma}^{(2)} \Delta q_{\alpha} \Delta q_{\beta} \Delta q_{\gamma}+\frac{1}{4} K_{\alpha \beta \gamma \delta}^{(3)} \Delta q_{\alpha} \Delta q_{\beta} \Delta q_{\gamma} \Delta q_{\delta}-\Delta Q_{\alpha} \Delta q_{\alpha}
$$

where

$$
\begin{aligned}
& K_{\alpha \beta}^{(1)}=\sum_{e=1}^{E} \int_{\Omega_{e}}\left\{C_{i j k l} B_{i j \alpha} B_{k l \beta}+\tilde{\sigma}_{i j} \phi_{\alpha k, i} \phi_{\beta l, j}\right\} d \Omega, \\
& K_{\alpha \beta \gamma}^{(2)}=\sum_{e=1}^{E} \int_{\Omega_{e}}\left\{\frac{3}{2} C_{i j k l}\left(B_{i j \alpha} B_{k l \beta \gamma}+B_{i j \alpha \beta} B_{k l \gamma}\right)\right\} d \Omega, \\
& K_{\alpha \beta \gamma \delta}^{(3)}=\sum_{e=1}^{E} \int_{\Omega_{e}} 2 C_{i j k l} B_{i j \alpha \beta} B_{k l \gamma \delta} d \Omega,
\end{aligned}
$$

and also

$$
\Delta Q_{\alpha}=\sum_{e=1}^{E} \int_{\Omega_{e}} \rho \Delta f_{i} \phi_{\alpha i} d \Omega+\sum_{e=1}^{E} \int_{\partial \Omega_{e}} \Delta \hat{t}_{i} \phi_{\alpha i} d(\partial \Omega)
$$

Of course, the first order stiffness matrix consists of the constitutive, initial stress and initial displacement matrices, i.e.,

$$
K_{\alpha \beta}^{(l)}=K_{\alpha \beta}^{(e)}+K_{\alpha \beta}^{(\sigma)}+K_{\alpha \beta}^{(u)}
$$


Minimization of the functional given in Eq.(3.4) with respect to the displacements vector $\Delta q_{\alpha}$ leads to the statement

$$
K_{\alpha \beta}^{(1)} \Delta q_{\beta}+K_{\alpha \beta \gamma}^{(2)} \Delta q_{\beta} \Delta q_{\gamma}+K_{\alpha \beta \gamma \delta}^{(3)} \Delta q_{\beta} \Delta q_{\gamma} \Delta q_{\delta}=\Delta Q_{\alpha},
$$

which is most frequently solved in engineering practice in the reduced form

$$
\left(K_{\alpha \beta}^{(e)}+K_{\alpha \beta}^{(\sigma)}+K_{\alpha \beta}^{(u)}\right) \Delta q_{\beta}=\Delta Q_{\alpha}
$$

We solve numerically the linearized stability problem given by the following matrix equation (Kleiber, 1985; Kleiber and Hien, 1997):

$$
K_{\alpha \beta}^{(e)} q_{\beta}+\lambda\left(K_{\alpha \beta}^{(\sigma)}(\boldsymbol{\sigma})+K_{\alpha \beta}^{(u)}(\boldsymbol{q})\right) q_{\beta}=0
$$

where the pair $(\boldsymbol{\sigma}, \boldsymbol{q})$ are the stresses and displacements obtained for the linear problem under the external load $Q_{\alpha}$; the load multiplier $\lambda$ is introduced as

$$
\lambda Q_{\alpha}=Q_{\alpha}+\mu \Delta Q_{\alpha}, \quad \lambda \boldsymbol{\sigma}=\boldsymbol{\sigma}+\mu \Delta \boldsymbol{\sigma}, \quad \lambda \boldsymbol{q}=\boldsymbol{q}+\mu \Delta \boldsymbol{q} .
$$

Further, $\mu$ and $\boldsymbol{q}$ are the eigenvalue and eigenvector corresponding to the bifurcation point, which is expressed by the following well known condition:

$$
\left\{K_{\alpha \beta}^{(e)} q_{\beta}+K_{\alpha \beta}^{(\sigma)}(\boldsymbol{\sigma})+K_{\alpha \beta}^{(u)}(\boldsymbol{q})\right\}+\mu\left\{K_{\alpha \beta}^{(\sigma)}(\Delta \boldsymbol{\sigma})+\Delta K_{\alpha \beta}^{(u)}(\boldsymbol{q}, \Delta \boldsymbol{q})\right\} q_{\beta}=0
$$

It simply follows the basic incremental equilibrium equation

$$
\left\{K_{\alpha \beta}^{(e)}+K_{\alpha \beta}^{(\sigma)}(\boldsymbol{\sigma})+K_{\alpha \beta}^{(u)}(\boldsymbol{q})\right\} \Delta q_{\beta}=\Delta Q_{\alpha}
$$

where the next possible load increment denoted by $\mu \Delta Q_{\alpha}$ induces extra stresses $\mu \Delta \boldsymbol{\sigma}$ and extra displacements $\mu \Delta \boldsymbol{q}$ such that

$$
\left\{K_{\alpha \beta}^{(e)}+K_{\alpha \beta}^{(\sigma)}(\boldsymbol{\sigma}+\mu \Delta \boldsymbol{\sigma})+K_{\alpha \beta}^{(u)}(\boldsymbol{q}+\mu \Delta \boldsymbol{q})\right\} \Delta q_{\beta}=\Delta Q_{\alpha} .
$$

We apply here Taylor expansion of the first order to these increments

$$
K_{\alpha \beta}^{(\sigma)}(\boldsymbol{\sigma}+\mu \Delta \boldsymbol{\sigma})=K_{\alpha \beta}^{(\sigma)}(\boldsymbol{\sigma})+\mu K_{\alpha \beta}^{(\sigma)}(\Delta \boldsymbol{\sigma})
$$

and

$$
K_{\alpha \beta}^{(u)}(\boldsymbol{q}+\mu \Delta \boldsymbol{q})=K_{\alpha \beta}^{(u)}(\boldsymbol{q})+\mu \Delta K_{\alpha \beta}^{(u)}(\boldsymbol{q}, \Delta \boldsymbol{q}),
$$


to linearize the last increment with respect to its arguments.

Traditionally, the Least Squares Method needs an iterative solution of the initial matrix equation around the expectation of the given input parameter and there holds here

$$
K_{\alpha \beta(i)}^{(e)} q_{\beta(i)}+\lambda_{(i)}\left(K_{\alpha \beta(i)}^{(\sigma)}(\boldsymbol{\sigma})+K_{\alpha \beta(i)}^{(u)}(\boldsymbol{q})\right) q_{\beta(i)}=0
$$

where $i=1, \ldots, n, \alpha, \beta=1, \ldots, N$. It results in a sequence of the pairs $\left(b_{i}, \lambda_{(i)}\right)$ used further to recover the polynomial response function for the critical load multiplier as in Kamiński (2013)

$$
\lambda_{c r}=\lambda_{c r}(b)=D^{(j)} b^{j}, \quad j=1, \ldots, m .
$$

It leads through the generalized stochastic perturbation technique to the up of the fourth order probabilistic characteristics (expectations, coefficients of variation, skewnesses and kurtosis) of this multiplier. Computer analysis includes three studies - first we postpone last two stiffness matrices in Eq.(3.16), then we drop off the last matrix by only and finally, we solve this equation including all its components.

We consider a residual in a given trial point indexed by $i$ as a difference between this trial value and a predicted curve to get the Weighted Least Squares Method (WLSM) matrix formulation relevant to the stability analysis with uncertain parameters, i.e.,

$$
r_{(i)}=\lambda_{c r(i)}-f\left(b_{i}, D^{(i)}\right) .
$$

Determination of the coefficients $D^{(i)}$ proceeds by using of the following gradient equations system:

$$
\frac{\partial\left(\sum_{i=1}^{n} r_{(i)}^{2}\right)}{\partial D^{(j)}}=2 \sum_{i=1}^{n} \frac{r_{(i)} \partial r_{(i)}}{\partial D^{(j)}} ; \quad j=1, \ldots, n
$$

Substituting Eq.(3.21) into Eq.(3.22) results in

$$
-2 \sum_{i=1}^{n} r_{(i)} \frac{\partial f\left(b_{i}, D^{(i)}\right)}{\partial D^{(j)}}=0 ; \quad j=1, \ldots, n
$$

Denoting here for the gradient $J_{i j}=\frac{\partial f\left(b_{i}, D^{(i)}\right)}{\partial D^{(j)}}$, we solve it for the coefficients $D^{(k)}$

$$
\sum_{i=1}^{n} \sum_{j=1}^{n} J_{i j} J_{i k} D^{(k)}=\sum_{i=1}^{n} J_{i j} \lambda_{c r(i)} ; \quad j=1, \ldots, n
$$

We modify this approach by the Aitken weighting procedure, where each trial point is associated to some parameter $w_{i i}$ such that the least squares functional equals to 


$$
S_{w}=\sum_{i=1}^{n} w_{i i} r_{(i)}^{2}
$$

Then, the LSM gradient becomes

$$
-2 \sum_{i=1}^{n} w_{i i} r_{(i)} \frac{\partial f\left(b_{i}, D^{(i)}\right)}{\partial D^{(j)}}=0 ; \quad j=1, \ldots, n
$$

Finally, the linear equations system to be solved yields

$$
\sum_{i=1}^{n} \sum_{j=1}^{n} J_{i j} w_{i i} J_{i k} D^{(k)}=\sum_{i=1}^{n} w_{i i} J_{i j} \lambda_{c r(i)} ; \quad j=1, \ldots, n
$$

In further computational experiments we use (a) the uniform weights equal 1 within the entire interval of trial values, (b) triangular distribution of the weights having maximum at the expectation in the middle of this interval and minimum values at both ends and finally, (c) the Dirac-like distribution, where the largest weight corresponds to the input expectation and unit weight is adopted elsewhere. The key question is whether the weighting process increases probabilistic convergence of the stochastic perturbation technique and the overall quality and stability of the results for the increasing value of the input coefficient of variation or not.

The final procedure is a symbolic calculation of the probabilistic moments of the critical load multiplier. It proceeds using the general order Taylor expansion of a stability limit $\lambda_{c r}$ with respect to the input Young modulus $e$ (Kamiński, 2013; Kamiński and Solecka, 2013; Kamiński and Strąkowski, 2013)

$$
\lambda_{c r}=\lambda_{c r}^{0}+\left.\sum_{i=1}^{n} \frac{\varepsilon^{i}}{i !} \frac{\partial^{i} \lambda_{c r}}{\partial e^{i}}\right|_{e=e^{0}} \Delta e^{i}
$$

where the order $n$ is smaller than the polynomial order provided in Eq.(3.20). It is inserted into successive equations for the basic probabilistic moments and characteristics of limit $\lambda_{c r}$ itself. We show as an illustration the expected values; there holds

$$
E\left[\lambda_{c r}\right]=\int_{-\infty}^{+\infty} \lambda_{c r}(e) p_{e}(x) d x=\int_{-\infty}^{+\infty}\left\{\lambda_{c r}^{0}+\left.\sum_{i=1}^{n} \frac{\varepsilon^{i}}{i !} \frac{\partial^{i} \lambda_{c r}}{\partial e^{i}}\right|_{e=e^{0}} \Delta e^{i}\right\} p_{e}(x) d x
$$

Putting in above $\varepsilon=1$ (Kleiber and Hien, 1992) and using the definitions of the central probabilistic moments of the variable $e$ one can get here

$$
\begin{aligned}
& E\left[\lambda_{c r}\right]=\int_{-\infty}^{+\infty} \lambda_{c r}^{0}(e) p_{e}(x) d x+\left.\int_{-\infty}^{+\infty} \sum_{i=1}^{n} \frac{\varepsilon^{i}}{i !} \frac{\partial^{i} \lambda_{c r}}{\partial e^{i}}\right|_{e=e^{0}} \Delta e^{i} p_{e}(x) d x= \\
& =\left.\lambda_{c r}^{0}\right|_{e=e^{0}}+\left.\sum_{i=1}^{n} \frac{\varepsilon^{i}}{i !} \frac{\partial^{i} \lambda_{c r}}{\partial e^{i}}\right|_{e=e^{0}} \mu_{i}(e)
\end{aligned}
$$

where all the moments of an odd order simply vanish here because of basic property of Gaussian variables 


$$
\mu_{i}(e)=\left\{\begin{array}{cc}
0, i=2 k+1, & k \in \aleph \\
\sigma^{i}(e)(i-1) ! !, & i=2 k
\end{array}\right.
$$

Analogous perturbation-based formulas for higher moments and coefficients may be found in Kamiński (2013), Kamiński and Solecka (2013), Kamiński and Strąkowski (2013), for instance.

\section{Computational experiments}

As a numerical illustration to this problem we consider the steel telecommunication tower $(60.0$ meters high) being a spatial structure with the rectangular cross-section consisting of ten segments, where up to the ninth segment (on the level of 54.0 meters) it has linear geometrical convergence and forms a prism with the dimensions $4.82 \mathrm{~m} \times 4.82 \mathrm{~m}$ at the base and $0.90 \mathrm{~m} \times 0.90 \mathrm{~m}$ at its top. The basic structural elements such as the edge beams and rebars are connected with bolts in lower segments and are welded at the two highest segments of this structure. Numerical analysis is provided using a combination of the structural analysis FEM system ROBOT (ROBOT Structural Analysis Professional 2011, User's Manual, 2010) and the computer algebra system MAPLE, where FEM discretization is built up with the use of 400 two-noded elastic truss 3D elements and 228 two-noded elastic 3D beam elements connected in 806 nodal points. The edge beams are designed with the round pipes of the diameters varying from $\varnothing 139.7 \mathrm{~mm} \times 8 \mathrm{~mm}$ until $\varnothing 60.3 \mathrm{~mm} \times 3 \mathrm{~mm}$; the rebars are taken as the cold formed C-bars (lower two segments), round pipes (segments at the middle of the tower) and full round cross-sections - the remaining upper cross-sections of this structure. The rebars are distributed according to the $\mathrm{X}$ pattern for 7 lower segments and in $K$ pattern for the last three segments. The detailed specification of the steel profiles and bolted connections is given in Tab.1 below, while their spatial distribution and photo of the structure are given in Fig.1. We notice there a steel ring with the external diameter equal to $3470 \mathrm{~mm}$ located at the level of 55.2 meters above the foundations, where two sector antennas cantilevers (about $1350 \mathrm{~mm}$ long) are attached. We account for the dead loads, technological installation weight as well as the wind pressure here. The critical load multiplier is added to four unit concentrated forces acting downwards and distributed symmetrically on the top steel ring at the ends of antennas cantilevers.

Table1. Structural steel profiles and connections in the telecommunication tower.

\begin{tabular}{|c|c|c|c|}
\hline Segment no. & Legs profiles $[\mathrm{mm}]$ & Rebars profiles $[\mathrm{mm}]$ & Bolts $[\mathrm{mm}]$ \\
\hline $\begin{array}{c}10 \\
\text { (tower top) }\end{array}$ & RO $60.3 \times 3.6$ & $\phi 22$ & M16 (class 8.8) \\
\hline 9 & RO $70.0 \times 4.0$ & RK $25.0 \times 3.0$ & M20 (8.8) \\
\hline 8 & RO $76.1 \times 5.0$ & RO $38.0 \times 4.0$ & M20/M12 (8.8) \\
\hline 7 & RO $88.9 \times 6.3$ & C $30 \times 30 \times 3$ & M20/M12/M18 (8.8) \\
\hline 6 & RO $88.9 \times 6.3$ & C $30 \times 30 \times 3$ & M20/M12/M18 (8.8) \\
\hline 5 & RO $114.3 \times 6.3$ & C $40 \times 40 \times 3$ & M22/M12 (8.8) \\
\hline 4 & RO $114.3 \times 6.3$ & C $40 \times 40 \times 3$ & M22/M12 (8.8) \\
\hline 3 & RO $139.7 \times 6.3$ & C $50 \times 50 \times 4$ & M24/M16 (8.8) \\
\hline 2 & RO $139.7 \times 8.0$ & C $50 \times 50 \times 4$ & M24/M16(8.8) \\
\hline 1 (tower base) & RO $139.7 \times 8.0$ & C 50 $50 \times 4$ & M24/M16 (8.8) \\
\hline
\end{tabular}



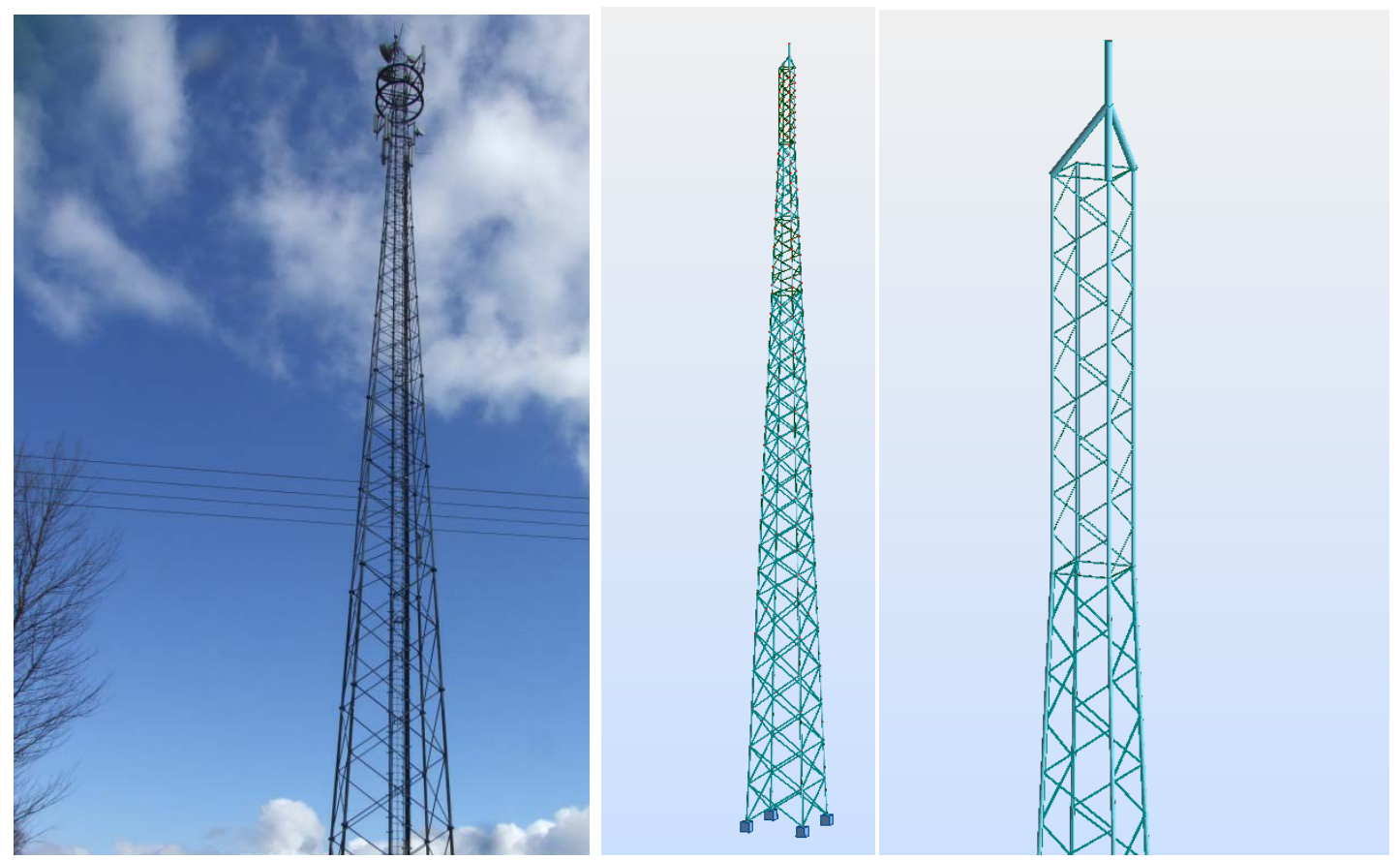

Fig.1. Photo (left), discretization of the entire structure (middle) and its top (right) in the FEM system ROBOT.

We use steel S355 for all the structural members with the Young modulus defined as the input random variable distributed according to the Gaussian probability function with the expected value equal to $e=210 \mathrm{GPa}$ (Poisson ratio $v$ equals 0.3 , of course), where its coefficient of variation $\alpha$ is an additional design parameter of the computational example. We use eleven trial points to recover the response functions between the critical value and the Young modulus and these are 189.0, 193.2, 197.4, 201.6, 205.8, 210.0, 214.2, 218.4, 222.6, 226.8, 231.0 GPa. The Finite Element Method analysis includes the following three different case studies: (a) initial stiffness matrix (composed using the elastic part only) with the corresponding critical values given in the left graphs, (b) including extra stiffness fluctuation from the longitudinal forces on the deformed elements (middle graphs of the probabilistic moments) and (c) including finally the stresses variations resulting from the computed deformations (right series of the next graphs). We account for maximum ten critical values of the engineering structure examined, numerical error during determination of the critical values is defined as equal to 0.0001 , iteration number equal to 40 , while the eigenproblem has been entirely solved using the subspace iteration algorithm. A nonlinear stability analysis (of the second and of the third order) has been carried out via the incremental approach using 5 load increments, 40 iterations for each load increment, maximum 3 reductions of a single increment, coefficient of the increment length taken as 0.5 and tolerance of the residual forces equal to 0.0001 has been adopted.

The resulting probabilistic moments and coefficients - expectations, coefficients of variation, skewness and kurtosis - are shown in Figs 2-13 for the first critical value and in Figs 14-24 - for the fifth critical load multiplier. This visualization is provided for each probabilistic characteristics in three independent rows - first for the unweighted LSM (Figure 2 for the first eigenvalue expectation - weights distribution is taken as the set $[1,1,1,1,1,1,1,1,1,1,1])$, then for the triangular weighting scheme $[1,2,3,4,5,6,5,4,3,2,1]$ (Figure 3 for this case) and finally, with the use of the Dirac-type weights distribution $[1,1,1,1,1,6,1,1,1,1,1]$ (Fig.4). These functions are presented also with respect to the order of stochastic perturbation technique, see Fig.2, for instance, as well as in addition to the approximating polynomial order, see Figs 3-4, for example, where both odd and even order terms are included. We contrast probabilistic moments of the first critical value against these adjacent to the fifth one to verify any possible qualitative and quantitative differences in-between such characteristics for various forms of the stability loss (in 
compression, bending and combined bending-twisting admissible for the structures of similar structural morphology).
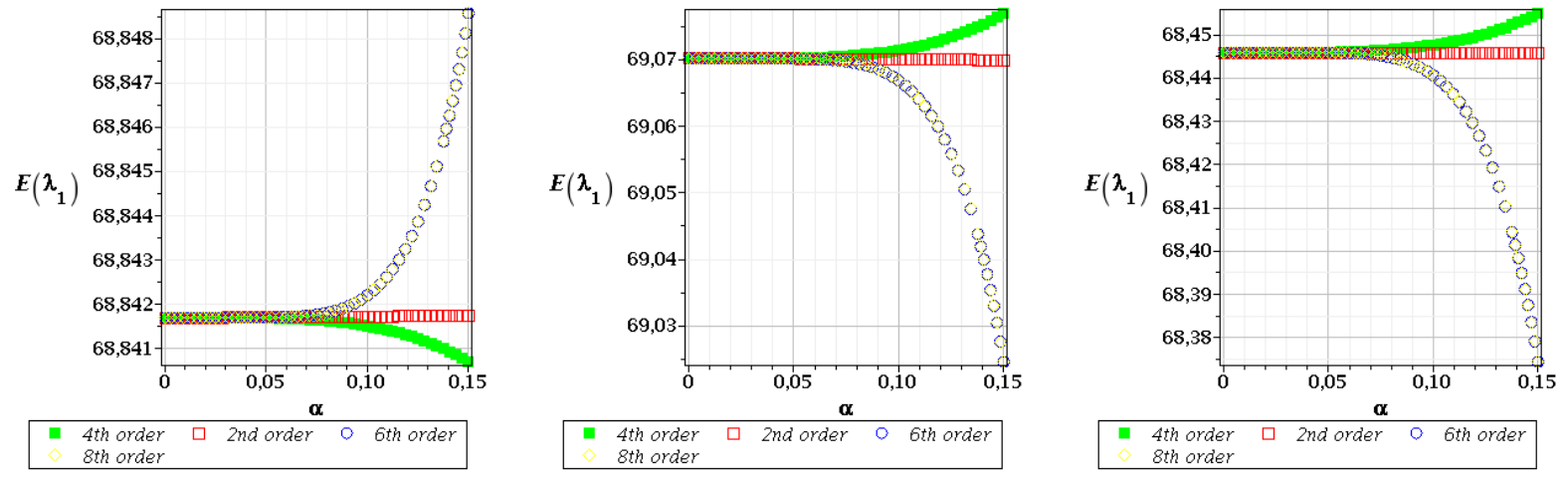

Fig.2. Expected values for the first critical value in the unweighted LSM approach $\left(1^{\text {st }}\right.$ order -1 eft, $2^{\text {nd }}$ order - middle, $3^{\text {rd }}$ order - right graph).
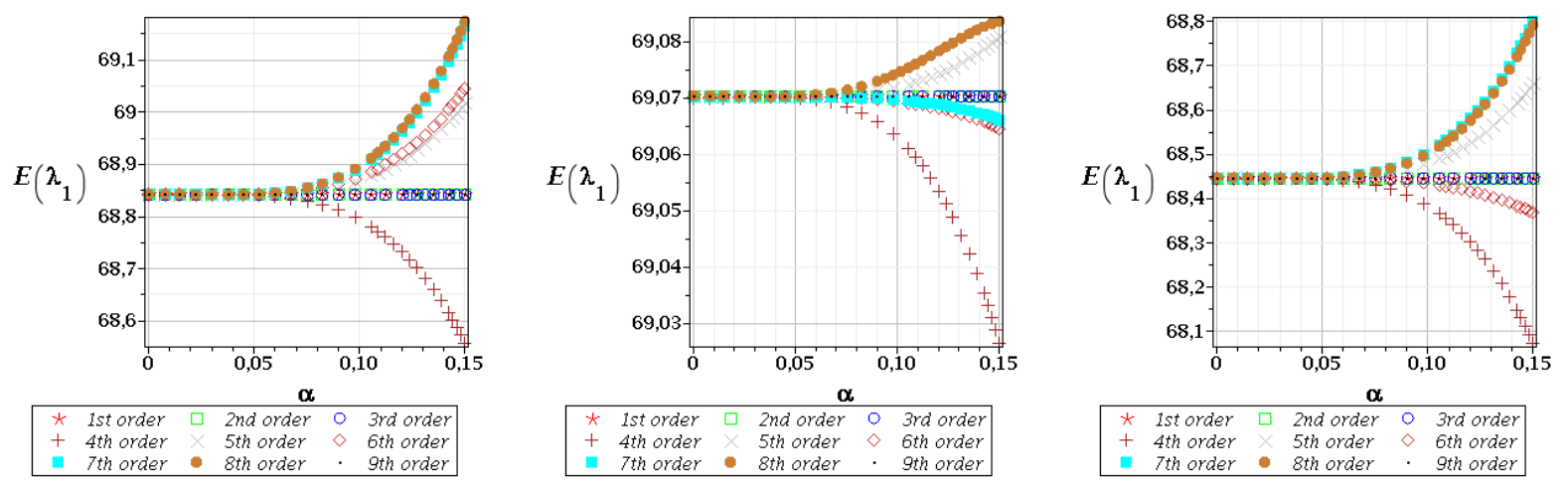

Fig.3. Expected values for the first critical value in the triangular weighted LSM approach $\left(1^{\text {st }}\right.$ order - left, $2^{\text {nd }}$ order - middle, $3^{\text {rd }}$ order - right graph).
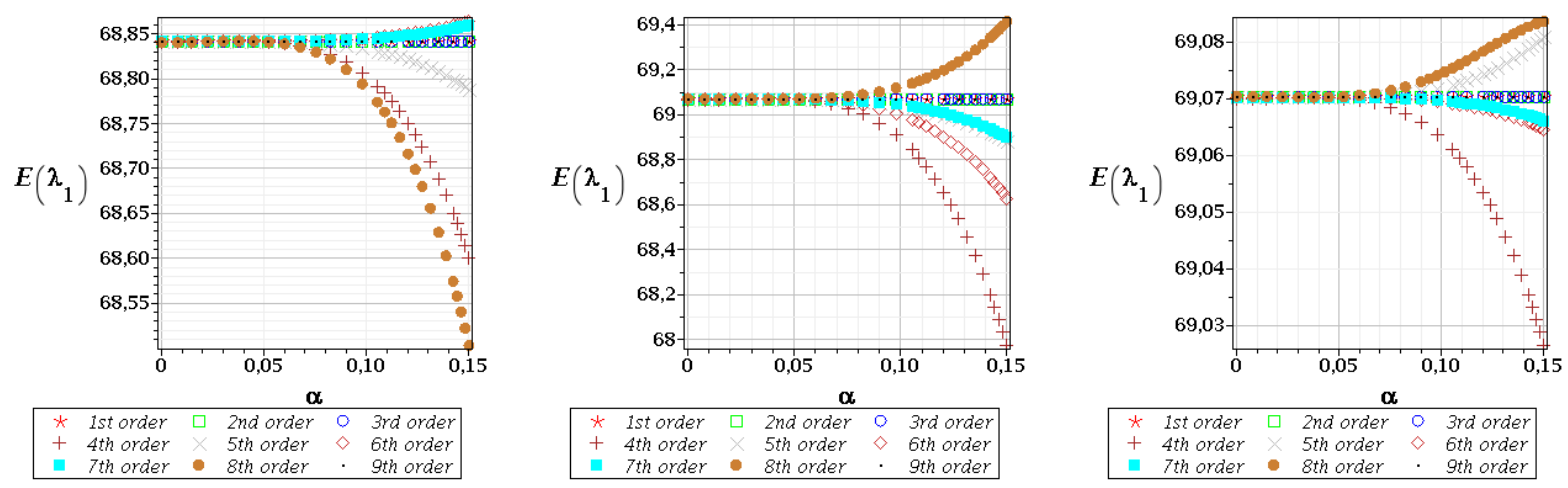

Fig.4. Expected values for the first critical value in the Dirac weighted LSM approach $\left(1^{\text {st }}\right.$ order - left, $2^{\text {nd }}$ order - middle, $3^{\text {rd }}$ order - right graph). 



Fig.5. Coefficients of variation for the first critical value in the unweighted LSM approach $\left(1^{\text {st }}\right.$ order - left, $2^{\text {nd }}$ order - middle, $3^{\text {rd }}$ order - right graph).
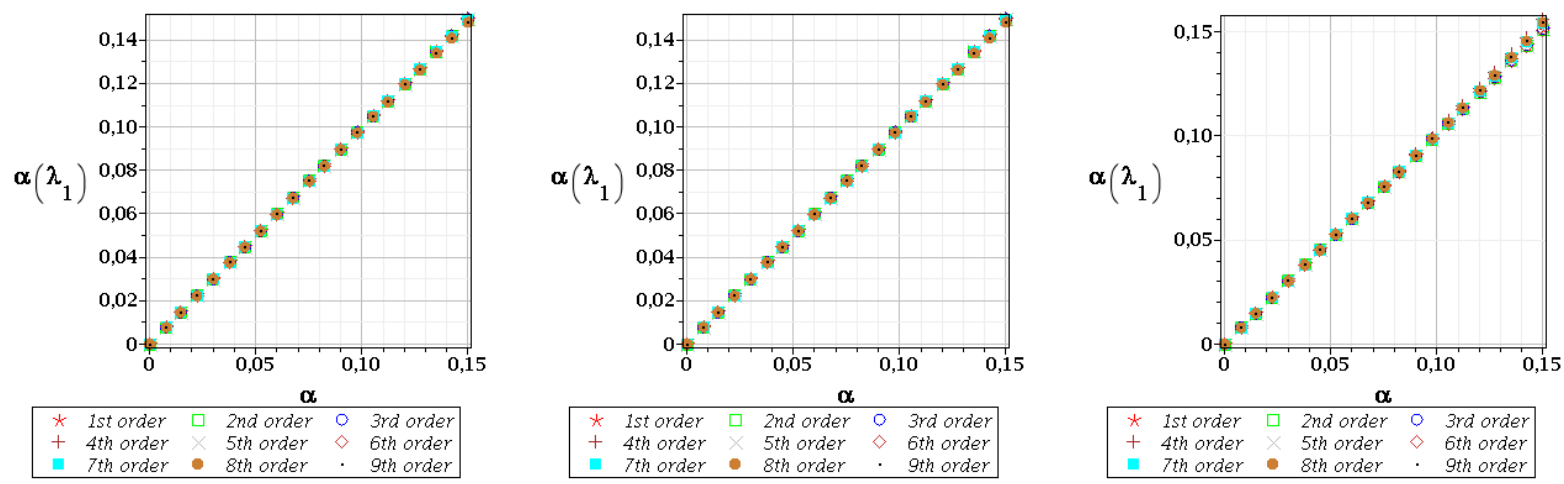

Fig.6. Coefficients of variation for the first critical value in the triangular weighted LSM approach $\left(1^{\text {st }}\right.$ order - left, $2^{\text {nd }}$ order - middle, $3^{\text {rd }}$ order - right graph $)$.


Fig.7. Coefficients of variation for the first critical value in the Dirac weighted LSM approach $\left(1^{\text {st }}\right.$ order left, $2^{\text {nd }}$ order - middle, $3^{\text {rd }}$ order - right graph). 

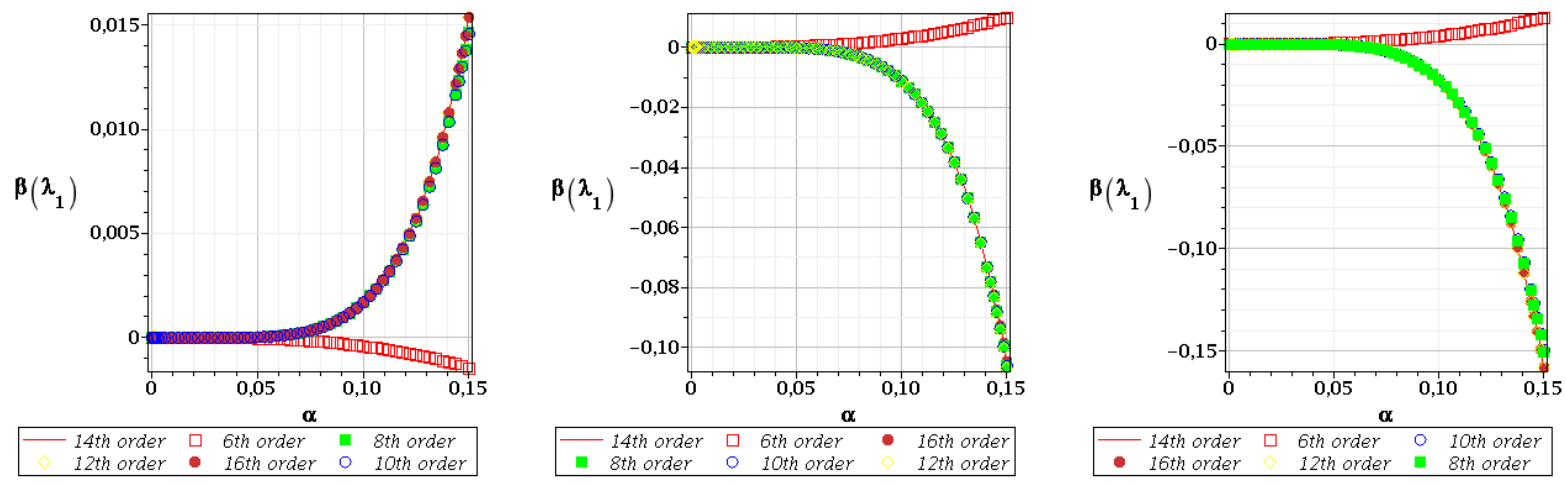

Fig.8. Skewness for the first critical value in the unweighted LSM approach $\left(1^{\text {st }}\right.$ order -1 eft, $2^{\text {nd }}$ order middle, $3^{\text {rd }}$ order - right graph).
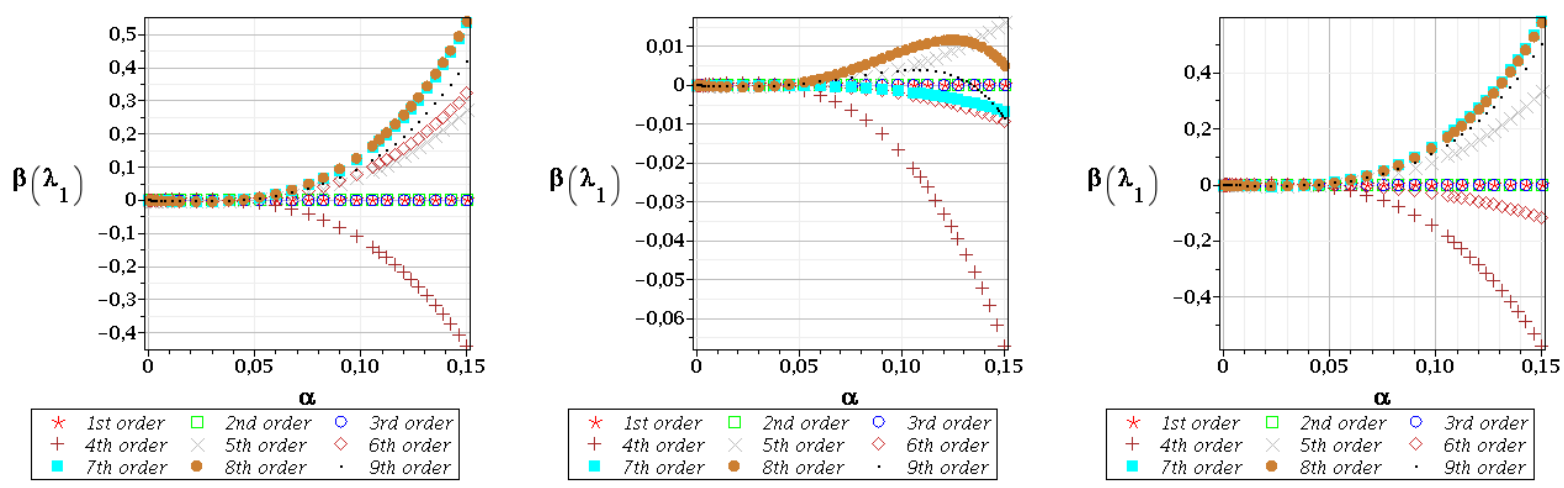

Fig.9. Skewness for the first critical value in the triangular weighted LSM approach $\left(1^{\text {st }}\right.$ order -1 eft, $2^{\text {nd }}$ order - middle, $3^{\text {rd }}$ order - right graph).


Fig.10. Skewness for the first critical value in the Dirac weighted LSM approach $\left(1^{\text {st }}\right.$ order - left, $2^{\text {nd }}$ order middle, $3^{\text {rd }}$ order - right graph). 

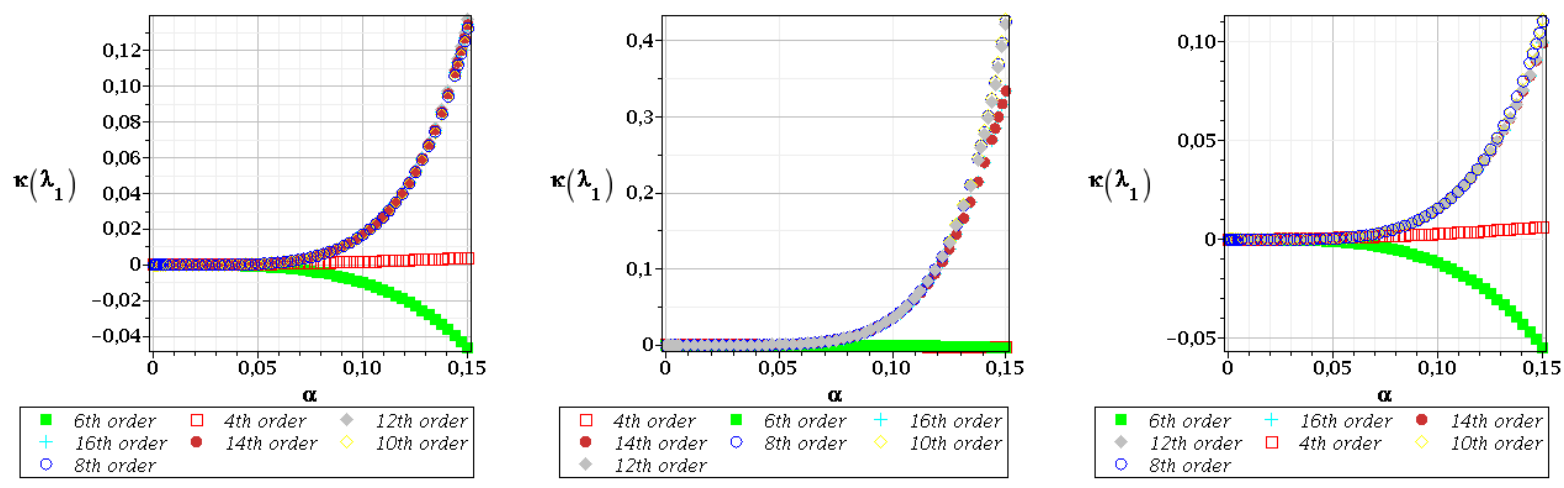

Fig.11. Kurtosis for the first critical value in the unweighted LSM approach $\left(1^{\text {st }}\right.$ order -1 eft, $2^{\text {nd }}$ order middle, $3^{\text {rd }}$ order - right graph).
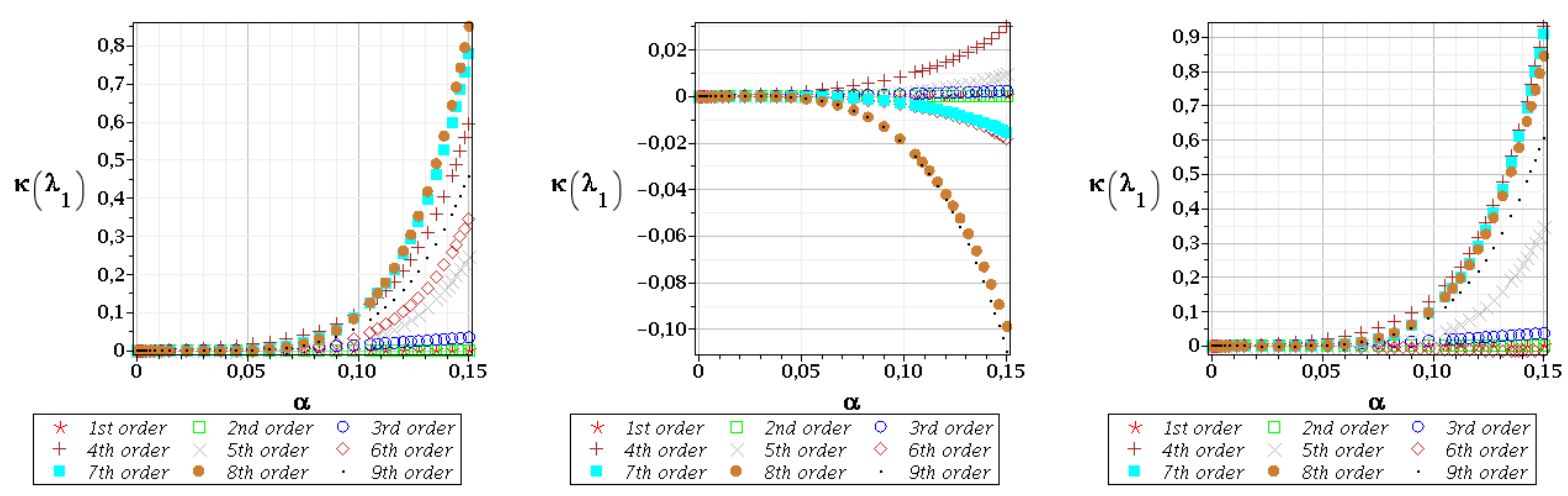

Fig.12. Kurtosis for the first critical value in the triangular weighted LSM approach $\left(1^{\text {st }}\right.$ order - left, $2^{\text {nd }}$ order - middle, $3^{\text {rd }}$ order - right graph).
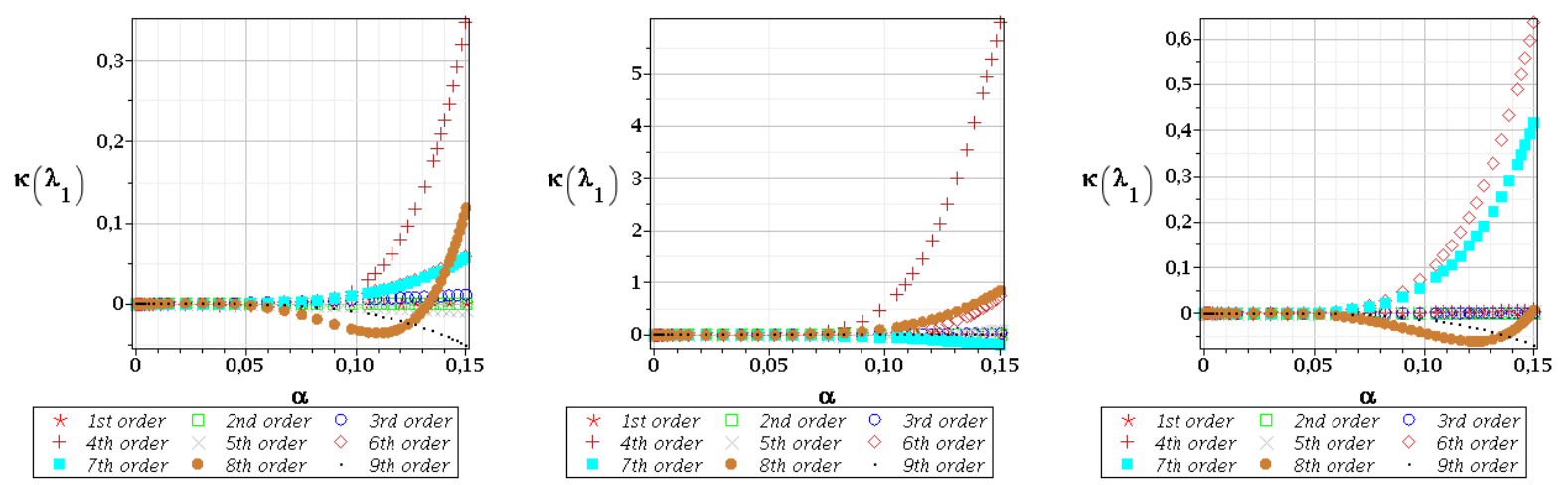

Fig.13. Kurtosis for the first critical value in the Dirac weighted LSM approach $\left(1^{\text {st }}\right.$ order - left, $2^{\text {nd }}$ order middle, $3^{\text {rd }}$ order - right graph).

A comparison of Figs 2-4 with 14-16 shows that the weighting procedure does not influence significantly probabilistic convergence of the stochastic perturbation technique as it was noticed for some elasticity problems before (Kamiński, 2013). The results are perfectly independent from an input coefficient of variation for all case studies in the interval $\alpha \in[0.0,0.075]$ and diverge further. However, the relative differences obtained for larger values are really negligible as they are smaller than $1 \%$ of the initial 
expectation. The largest values of the critical forces are noticed for the triangular distribution of the weights (as far as the weighting procedure is analyzed) and, independently, when elastic and stress stiffness matrices are inserted into the eigenproblem solution. The coefficients of variation (Figs 5-7 and Figs 17-19) are practically insensitive to the weighting scheme at all for both the first and fifth critical value (the same intervals and extreme values are obtained even for a various composition of the stiffness matrix of the system).

Considering the fact that the third and fourth order probabilistic moments need longer Taylor expansion to preserve satisfactory accuracy, we propose the 16th order perturbation analysis (Figs 8 and 11 for the first critical value), contrary to the first two approaches, where up to the 10th order expansions were preferred. A general observation is that these coefficients diverge probabilistically out of the interval $\alpha \in[0.0,0.075]$, while inside it we obtain 0 independent of the order of both perturbation and the polynomial response function. We obtain both positive and negative values of these coefficients in the same weighting scheme and different stiffness components (graphs in the same row) as well as for the same stiffness and various weighting procedures (graphs in the same columns). Extreme values of skewness and kurtosis are obtained here in the tenths and hundredths (close to the values characteristic for the Gaussian distribution). Once more, the weighting procedure has no apparent clear influence on the final results and, especially, their tendencies with increasing coefficient of variation of Young modulus for steel.


Fig.14. Expected values for the fifth critical value in the unweighted LSM approach $\left(1^{\text {st }}\right.$ order - left, $2^{\text {nd }}$ order - middle, $3^{\text {rd }}$ order - right graph).
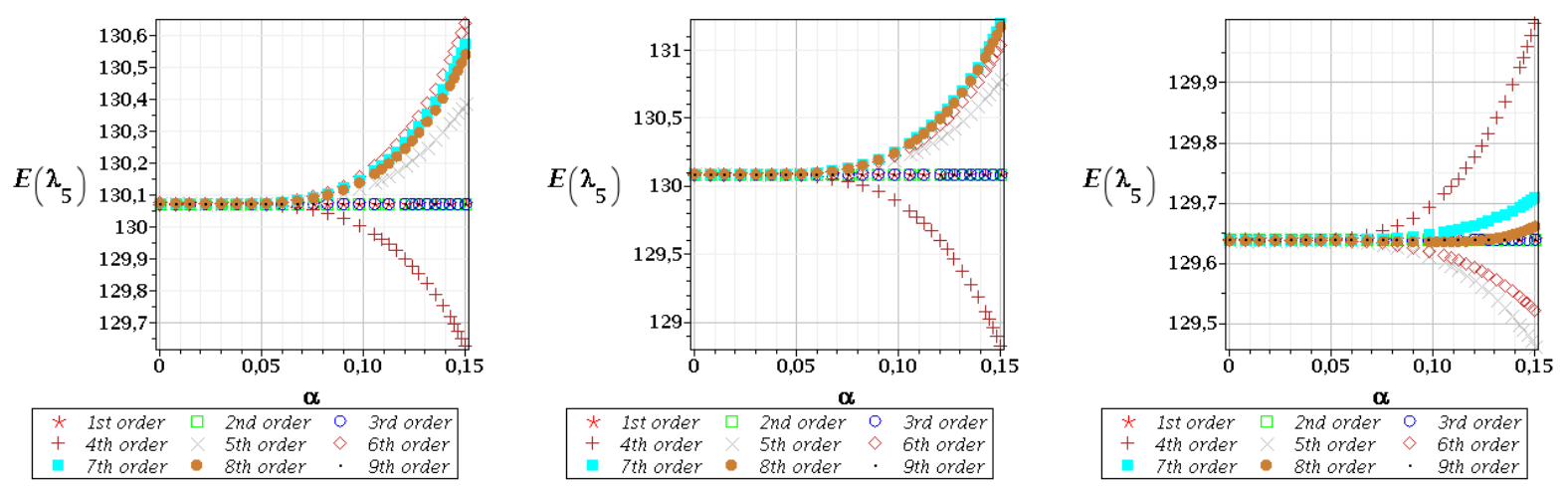

Fig.15. Expected values for the fifth critical value in the triangular weighted LSM approach $\left(1^{\text {st }}\right.$ order - left, $2^{\text {nd }}$ order - middle, $3^{\text {rd }}$ order - right graph). 



Fig.16. Expected values for the fifth critical value in the Dirac weighted LSM approach $\left(1^{\text {st }}\right.$ order - left, $2^{\text {nd }}$ order - middle, $3^{\text {rd }}$ order - right graph).
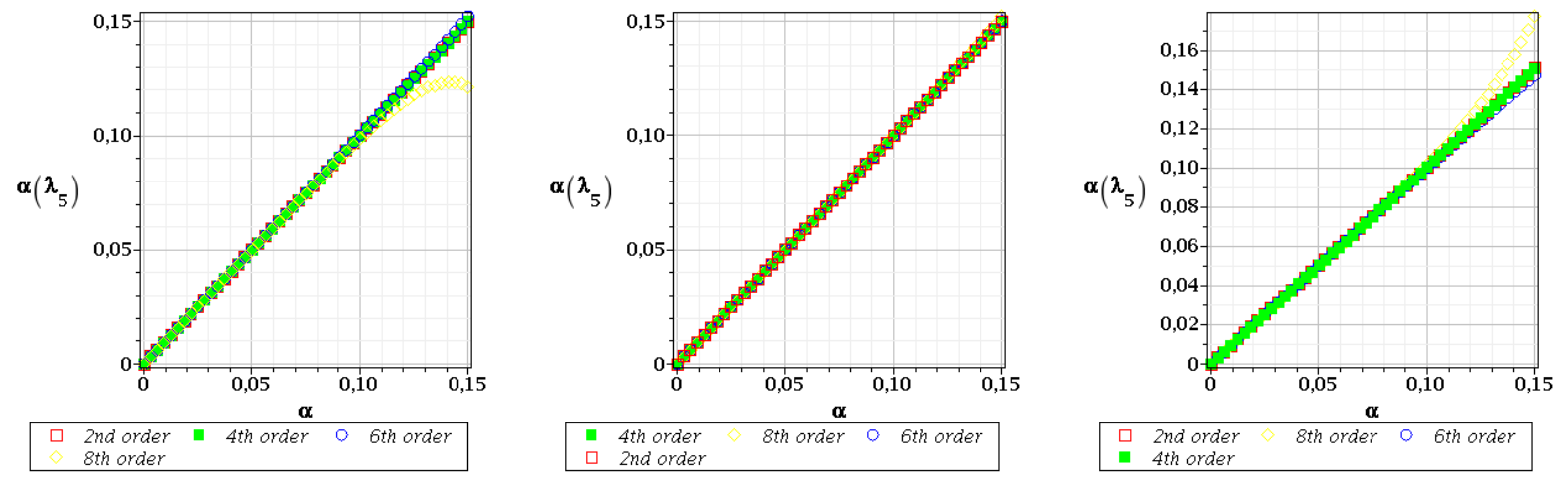

Fig.17. Coefficients of variation for the fifth critical value in the unweighted LSM approach ( $1^{\text {st }}$ order - left, $2^{\text {nd }}$ order - middle, $3^{\text {rd }}$ order - right graph).
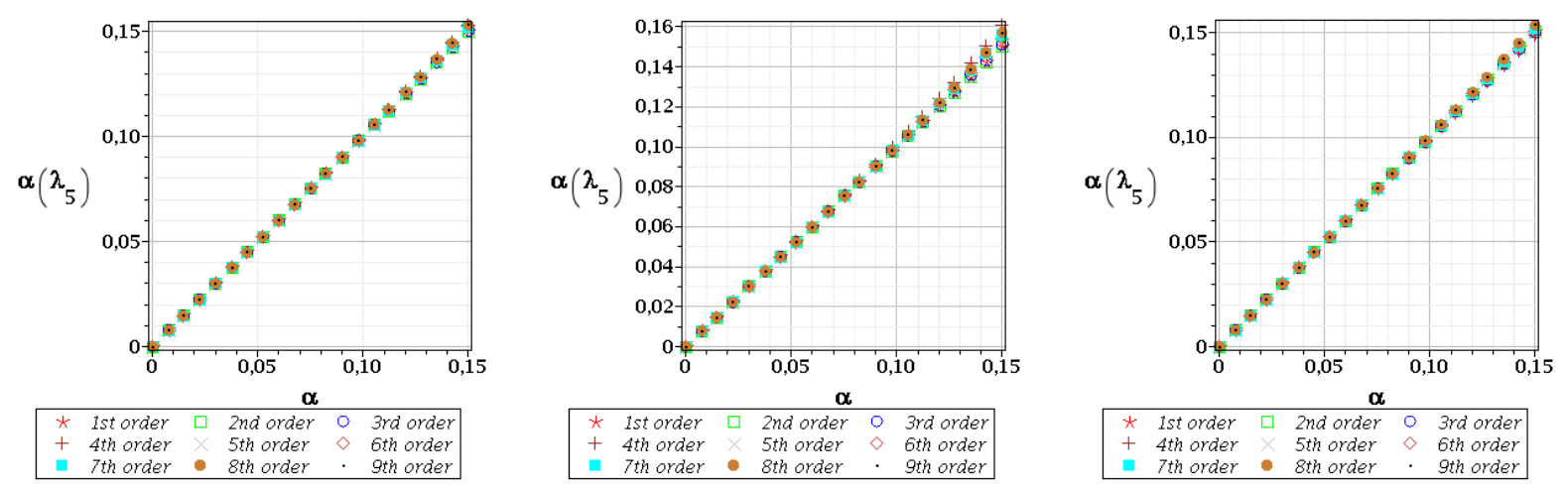

Fig.18. Coefficients of variation for the fifth critical value in the triangular weighted LSM approach $\left(1^{\text {st }}\right.$ order - left, $2^{\text {nd }}$ order - middle, $3^{\text {rd }}$ order - right graph $)$. 

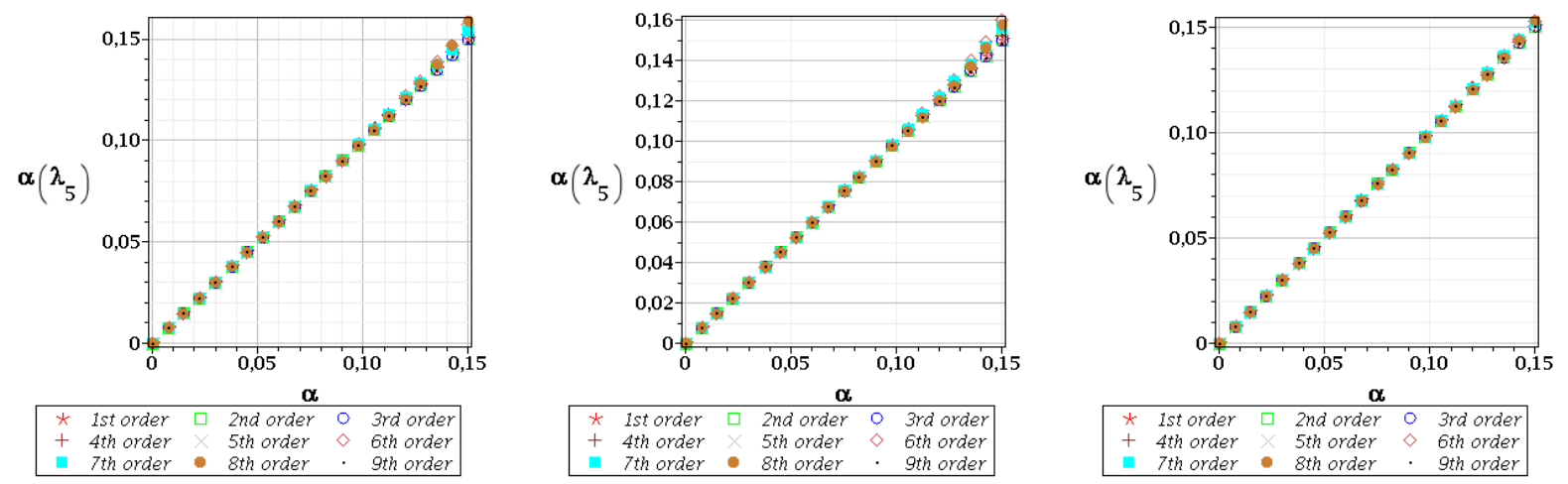

Fig.19. Coefficients of variation for the fifth critical value in the Dirac weighted LSM approach $\left(1^{\text {st }}\right.$ order left, $2^{\text {nd }}$ order - middle, $3^{\text {rd }}$ order - right graph).
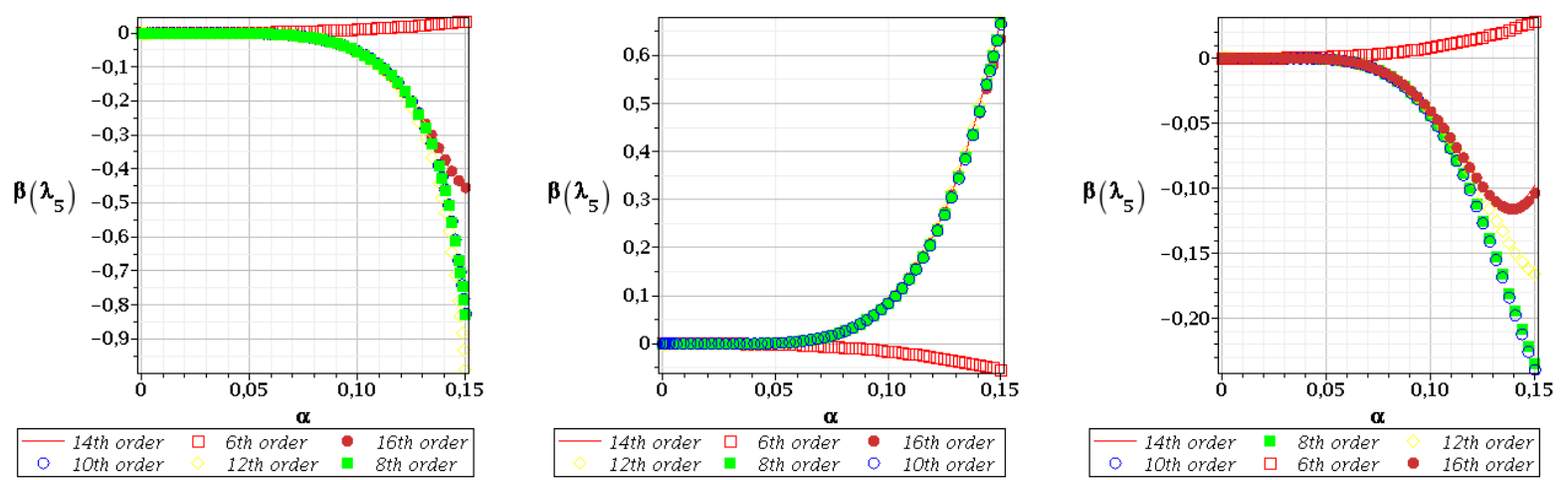

Fig.20. Skewness for the fifth critical value in the unweighted LSM approach $\left(1^{\text {st }}\right.$ order - left, $2^{\text {nd }}$ order middle, $3^{\text {rd }}$ order - right graph).
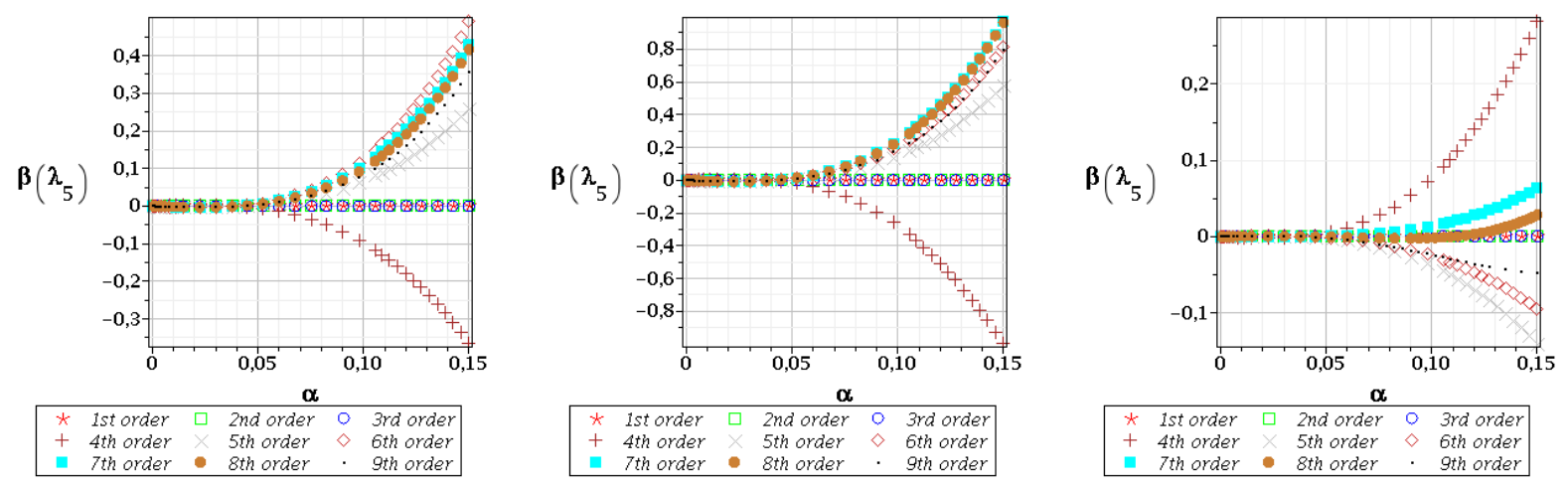

Fig.21. Skewness for the fifth critical value in the triangular weighted LSM approach $\left(1^{\text {st }}\right.$ order -1 left, $2^{\text {nd }}$ order - middle, $3^{\text {rd }}$ order - right graph). 

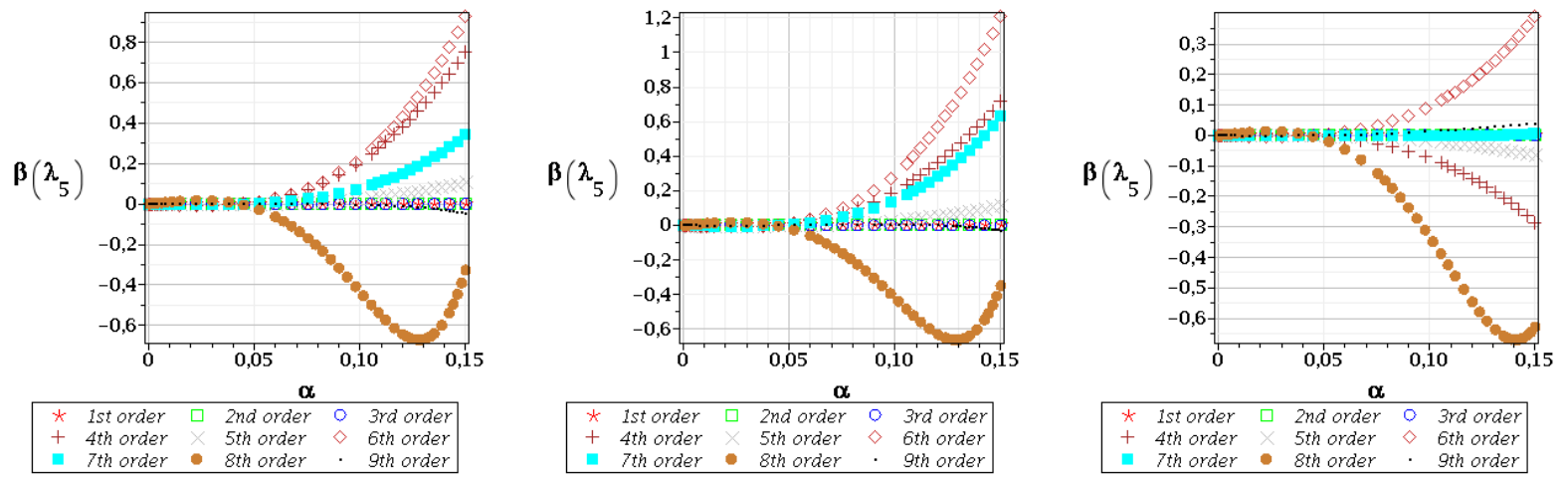

Fig.22. Skewness for the fifth critical value in the Dirac weighted LSM approach $\left(1^{\text {st }}\right.$ order - left, $2^{\text {nd }}$ order middle, $3^{\text {rd }}$ order - right graph).


Fig.23. Kurtosis for the fifth critical value in the unweighted LSM approach $\left(1^{\text {st }}\right.$ order -1 left, $2^{\text {nd }}$ order middle, $3^{\text {rd }}$ order - right graph).
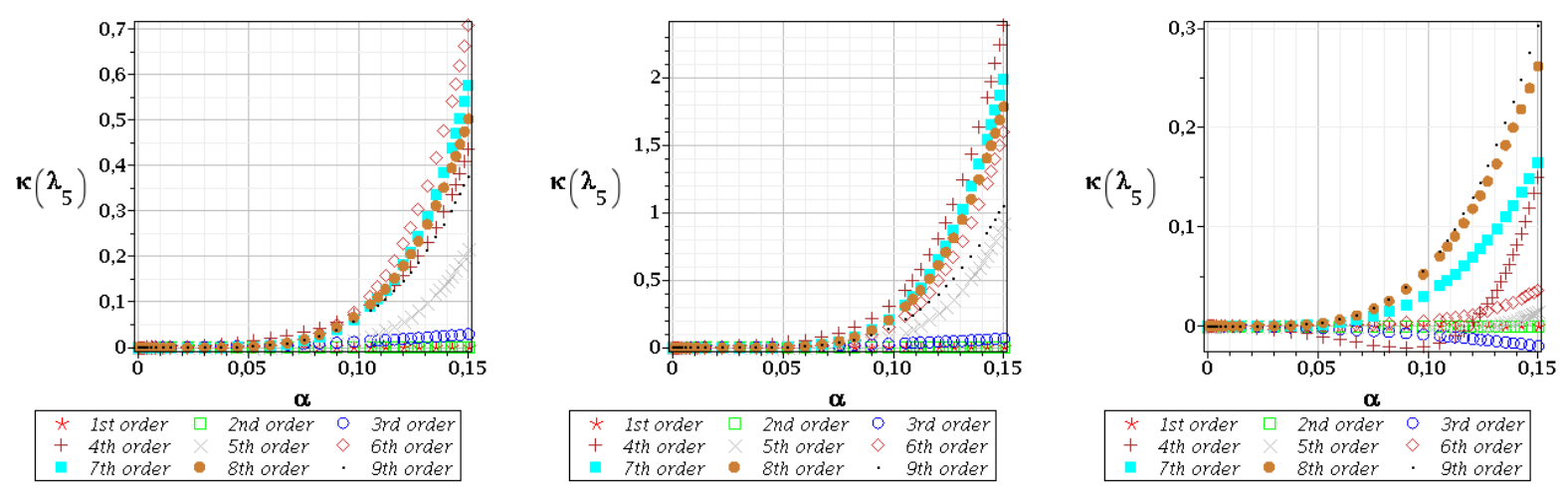

Fig.24. Kurtosis for the fifth critical value in the triangular weighted LSM approach $\left(1^{\text {st }}\right.$ order - left, $2^{\text {nd }}$ order - middle, $3^{\text {rd }}$ order - right graph). 

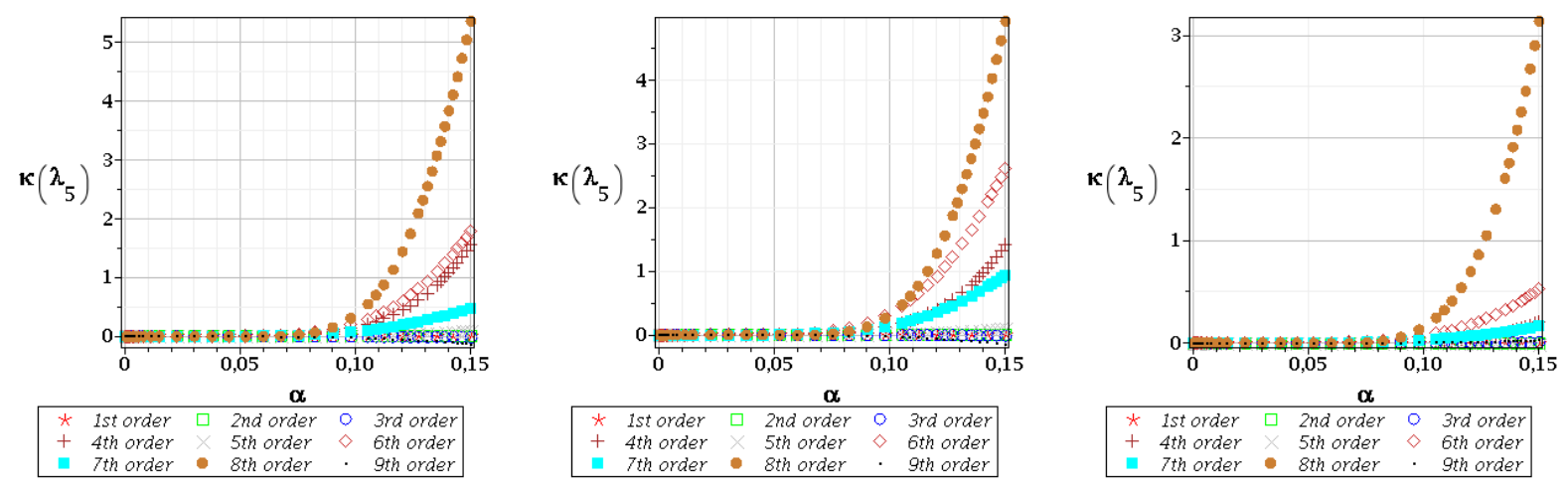

Fig.25. Kurtosis for the fifth critical value in the Dirac weighted LSM approach $\left(1^{\text {st }}\right.$ order -1 eft, $2^{\text {nd }}$ order middle, $3^{\text {rd }}$ order - right graph).

Further, it is remarkable in Figs 8-10, 11-13 as well as in Figs 20-22 and 23-25 that for smaller values of the input coefficient of variation $\alpha$, the resulting skewnesses and kurtosis of the critical force are with a relatively small error equal to 0 , while for larger values $(\alpha>0.075)$ these differences diverge a little typically for the generalized stochastic perturbation technique. The resulting coefficient of variation for both the first (Figs 5-7) and the fifth critical load magnitude (Figs 17-19) is linearly dependent upon the input one; they have the same values with a negligibly small error, independently of the number of stiffness matrix components included into the stochastic eigenproblem solution. It makes it possible to confirm a linear interrelation between the stability limit and Young modulus of the steel skeletal tower, which proves the Gaussian probability distribution of the force equivalent to the bifurcation point. Since the first and the fifth critical values behave probabilistically in almost the same manner, we observe that the classical Euler formula together with probabilistic basic algebraic formulas may certainly serve in reliability assessment and simplified engineering calculations in that particular area. These conclusions are significantly affected neither by the stochastic perturbation order nor by the polynomial response function order, which guarantees satisfactory accuracy of both qualitative and quantitative results. A common additional observation is that probabilistic characteristics higher than the second one start to diverge for an input coefficient of variation larger than 0.15 . Some small numerical discrepancies are also noticed for the expectations, but overall differences are smaller than a single percent for the entire availability interval of the input coefficient of variation $\alpha(e)$.

\section{Concluding remarks}

1. The computational analysis provided in this paper shows with no doubt that the uncertain critical loading under consideration remains Gaussian when Gaussian Young modulus is taken as the input random variable. This conclusion comes from the fact that the output coefficient of variation is equal to the input one independently of the deterministic stability analysis type, order of a stochastic perturbation and the polynomial response function and of the fact that both skewness and kurtosis equal 0 with a negligible error. It means that the output critical force is a linear combination of the input randomness source, which practically recovers the Gaussian PDF here (Elishakoff, 1983). Further, it enables the usage of the Eulerlike formula (Timoshenko and Gere, 1961) in practical calculations for the steel tower as it is noticed for both first and fifth (higher) critical load value and should be examined further in addition to the inertia moment of the edge beams in such a tower. It is remarkable that this study obeys parameter sensitivity analysis of the critical load for the towers with respect to the Young modulus as the stochastic perturbation technique is based on the sensitivity gradients of the increasing orders (at least of the first and of the second order (Kleiber and Hien, 1997) and as such may be engaged straightforwardly to both deterministic and probabilistic optimization procedures. It is also apparent that the weighting procedure taken into account during the Least Squares Method recovery of the response functions for the critical load is practically of a marginal importance for the considered probabilistic characteristics.

2. The computational study presented in this work is a preliminary study for the full extended stability analysis and examination of the limit states in high steel telecommunication towers. One of the most important issues 
is the local analysis of the structural members, where shell elements with random thickness should be accounted for together with limited stiffness of welded and/or bolted connections (Hadianfard and Razani, 2003; Papadopoulos et al., 2009). Such an analysis could reflect stochastic corrosion usually having apparently local character (Sadovský and Drdácký, 2001). The second, very important issue is post-buckling of the steel towers, where computational determination of the post-buckling paths (Schafer and GrahamBrady, 2006; Steinböck et al., 2008), especially in the stochastic context (especially with the use of generalized stochastic perturbation technique) and in reliability analysis using FORM and SORM, can be of the paramount importance. Needless to say, analogous numerical analysis would be interesting in the field of aluminum telecommunication structures (Kamiński and Solecka, 2013).

\section{Nomenclature}

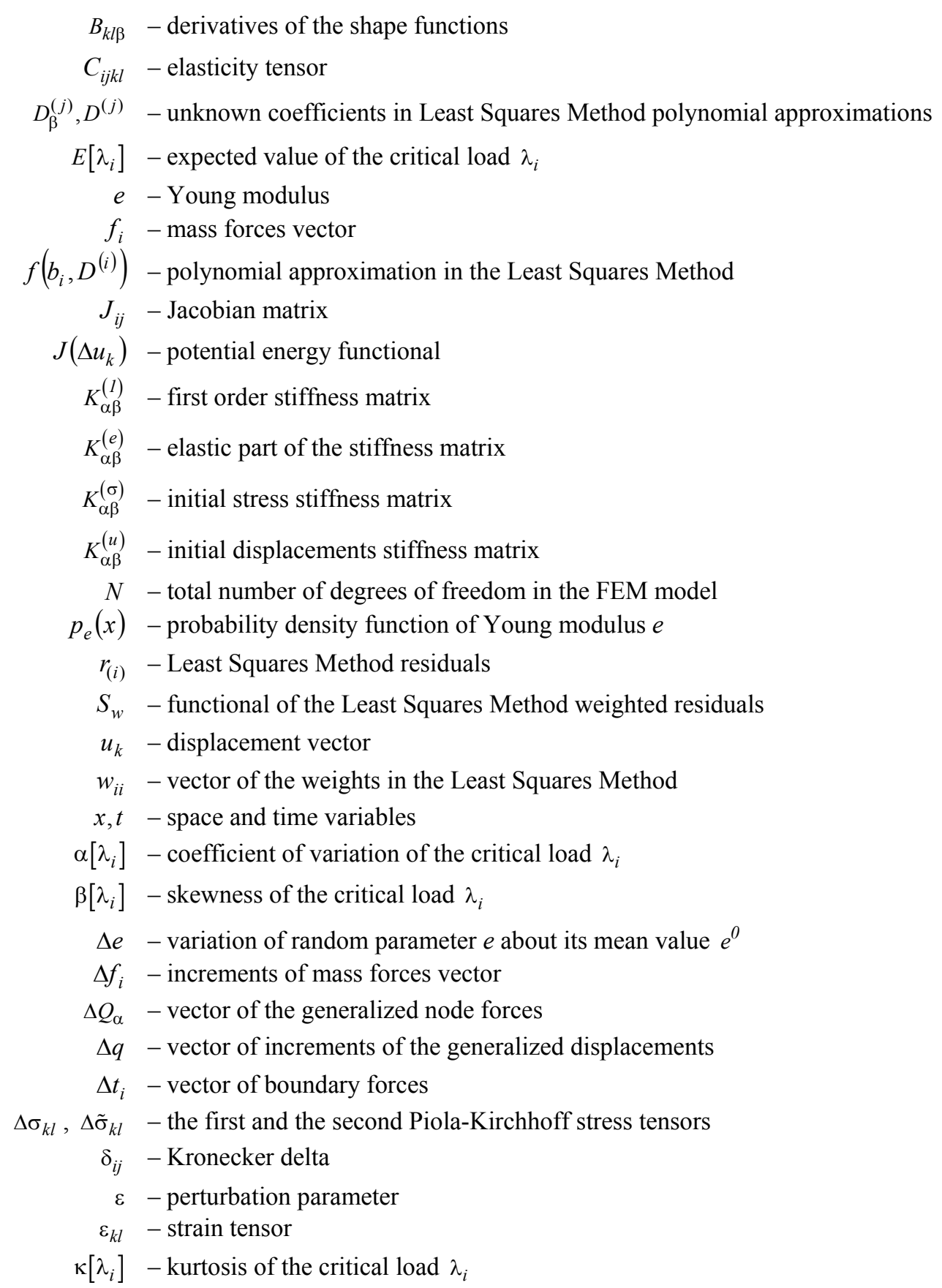


$\lambda_{c r}-$ critical load multiplier

$\lambda_{i}-i$-th critical load multiplier

$\mu_{i}(e)-i$-th central probabilistic moments of random parameter $e$

$v-$ Poisson ratio

$\rho-$ mass density

$\sigma_{k l}-$ stress tensor

$\sigma(e)$ - standard deviation of random parameter $e$

$\phi_{\alpha \beta}$ - shape function matrix

$\Omega$ - computational domain

$\partial \Omega_{\sigma}, \partial \Omega_{u}-$ external boundaries of $\Omega$ to define stress and displacements boundary conditions

$(.)^{0}-$ mean value of the parameter (.)

\section{References}

Elishakoff I. (1983): Probabilistic Methods in the Theory of Structures. - New York: Wiley-Interscience.

Elishakoff I. (2000): Uncertain buckling: its past, present and future. - International Journal of Solids and Structures, vol.37, pp.6869-6889.

Elishakoff I., Li Y.W. and Starnes J.H. (2001): Nonclassical Problems in the Theory of Elastic Stability. - Cambridge: Cambridge University Press.

Hadianfard M.A. and Razani R. (2003): Effects of semi-rigid behavior of connections in the reliability of steel frames. Journal of Structural Safety, vol.25, pp.123-138.

Kamiński M. (2013): The Stochastic Perturbation Method for Computational Mechanics. - Chichester: Wiley.

Kamiński M. and Solecka M. (2013). Optimization of the aluminium and steel telecommunication towers using the generalized perturbation-based Stochastic Finite Element Method. - Journal of Finite Elements Analysis and Design, vol.63, No.1, pp.69-79.

Kamiński M. and Strąkowski M. (2013): On the least squares stochastic finite element analysis of the steel skeletal towers exposed to the fire. - Archives of Civil and Mechanical Engineering, vol.13, pp.242-253.

Kleiber M. (1985): Finite Element Method in Nonlinear Continuum Mechanics (in Polish). - Warsaw-Poznań: Polish Scientific Publishers.

Kleiber M. and Hien T.D. (1992): The Stochastic Finite Element Method. - Chichester, Wiley.

Kleiber M. and Hien T.D. (1997): Parameter sensitivity of inelastic buckling and post-buckling response. - Computer Methods in Applied Mechanics and Engineering, vol.145, pp.239-262.

Melchers R.E. (1987): Structural Reliability. Analysis and Prediction. - Chichester: Ellis Horwood Limited.

Papadopoulos V., Stefanou G. and Papadrakakis M. (2009): Buckling analysis of imperfect shells with stochastic nonGaussian material and thickness properties. - International Journal of Solids and Structures, vol.46, pp.2800-2808.

ROBOT Structural Analysis Professional 2011, User's Manual (2010). Autodesk Inc.

Sadovský Z. and Drdácký M. (2001): Buckling of plate strip subjected to localised corrosion — a stochastic model. - J. Thin-Walled Struct. 39, pp.247-259.

Schafer B.W. and Graham-Brady L. (2006): Stochastic post-buckling of frames using Koiter's method. - International Journal of Structural Stability and Dynamics, vol.6, pp.333-358.

Steinböck, Jia X., Höfinger G., Rubin H. and Mang H.A. (2008): Remarkable postbuckling paths analyzed by means of the consistently linearized eigenproblem. - International Journal for Numerical Methods in Engineering, vol.76, pp.156-182.

Timoshenko S.P. and Gere J.M. (1961): Theory of Elastic Stability (second ed.). - New York: McGraw-Hill.

Received: March 24, 2014

Revised: January 26, 2015 